\title{
BMJ Open Feasibility and acceptability of study methods and psychosocial interventions for body image targeting women diagnosed with breast cancer: a protocol for a systematic review
}

\author{
Jennifer Brunet (D) , ${ }^{1}$ Jenson Price, ${ }^{1}$ Aurelie Baillot ${ }^{2}$
}

To cite: Brunet J, Price J, Baillot A. Feasibility and acceptability of study methods and psychosocial interventions for body image targeting women diagnosed with breast cancer: a protocol for a systematic review. BMJ Open 2021;11:e057309. doi:10.1136/ bmjopen-2021-057309

- Prepublication history and additional supplemental material for this paper are available online. To view these files, please visit the journal online (http://dx.doi.org/10.1136/ bmjopen-2021-057309).

Received 14 September 2021 Accepted 08 November 2021

Check for updates

(C) Author(s) (or their employer(s)) 2021. Re-use permitted under CC BY-NC. No commercial re-use. See rights and permissions. Published by BMJ.

${ }^{1}$ School of Human Kinetics, University of Ottawa, Ottawa, Ontario, Canada

${ }^{2}$ Département des sciences infirmières, Université du Québec en Outaouais, Gatineau, Québec, Canada

Correspondence to Dr Jennifer Brunet; Jennifer.Brunet@uottawa.ca

\section{ABSTRACT}

Introduction Improving body image may help to enhance the quality of life of women diagnosed with breast cancer. Although evidence suggests psychosocial interventions can effectively improve body image in this population, no review to date has assessed their feasibility or acceptability. This manuscript reports the protocol for a review summarising current evidence for the feasibility and acceptability of psychosocial interventions for body image targeting women diagnosed with breast cancer and the study methods used to evaluate the interventions in question to provide recommendations to optimise the success and sustainability of psychosocial interventions for body image and future studies. Results will also help to identify gaps in the existing evidence to provide direction for future research.

Methods and analysis We searched the following databases for articles published in the English language from January 2000 to June 2021 using a systematic search strategy: MEDLINE, Cumulative Index to Nursing and Allied Health Literature, Cochrane Central Register of Controlled Trials, PsychINFO and EMBASE. This search will be supplemented with a manual search of reference lists from relevant systematic reviews and included articles. Eligible studies will include peer-reviewed publications reporting on feasibility and acceptability in the evaluation of psychosocial interventions for body image targeting women diagnosed with breast cancer. All study designs are eligible, although articles are required to have reported on an intervention evaluation. Two reviewers will independently carry out study selection, extraction of quantitative and qualitative data and quality assessment. Data will be summarised in a narrative review and thematic analysis.

Ethics and dissemination No ethical approval is required because this is a protocol for a systematic review. On completion, results will be submitted for publication in a peer-reviewed scientific journal and for presentation at a relevant conference.

Trial registration This protocol has been registered in the Prospective Register of Systematic Reviews international registry (ID: CRD42021269062, 11 September 2021).
Strength and limitations of this study

- Search methods (eg, range of databases, search strategy) are comprehensive to ensure eligible studies are retrieved and included.

- All studies designs using quantitative, qualitative and mixed methods to evaluate an intervention are eligible to enable a broad and in-depth analysis of interventions.

- Data extraction will be conducted systematically using a piloted data extraction form and corresponding authors will be contacted for further details.

- The complexity of the concepts of 'feasibility' and 'acceptability', coupled with discrepancies in reporting and/or small sample sizes, may present challenges while interpreting and summarising data.

- The requirement for studies to be published in the English language in a peer-reviewed journal may result in a biased sample, publication bias and/or time-lag bias.

\section{INTRODUCTION}

According to the International Agency for Research on Cancer, ${ }^{1}$ there were an estimated 19.3 million new cancer cases and almost 10 million cancer deaths in 2020 globally. Breast cancer remains the most commonly diagnosed cancer in women, with an estimated 2.3 million new cases. ${ }^{1}$ Surgery, chemotherapy, radiation therapy and hormone therapy are considered the main treatment modalities for breast cancer and are used for most women. ${ }^{2}$ Such modalities can result in physical appearance changes (eg, breast(s) loss, scarring, alopecia, skin alterations, weight changes). Along with other side effects (eg, arm function impairment), physical appearance changes can have a substantial impact on body image, ${ }^{3}$ which in turn has been associated with reduced quality of life. ${ }^{34}$ Indeed, a review of reviews from 2008 to 2018 that focused on quality of life reveals that 
body image plays a key role in thwarting women's quality of life. ${ }^{5}$ Furthermore, visible physical appearance changes may lead to stigmatisation among women diagnosed with breast cancer, ${ }^{6-8}$ which has been associated with affective disorders (eg, depression), ${ }^{9}{ }^{10}$ and body image has been shown to meditate this association. ${ }^{8}$

Access to interventions aiming to improve body image during and after treatment is key to enhancing the quality of life in women diagnosed with breast cancer. ${ }^{11}$ In response, there has been a rapid increase in the number of studies delivering psychosocial interventions to women diagnosed with breast cancer with the purpose of improving body image. ${ }^{12} 13$ Reviews focusing on the effects of such interventions identified several approaches used (eg, psychoeducation, cognitive behavioural therapy, physical activity, art therapy, mindfulness, expressive writing) to improve body image. ${ }^{12-14}$ Promising evidence related to the improvement of body image was found for some interventions comprising psychotherapy, psychoeducation or physical activity in women diagnosed with breast cancer. ${ }^{12-14}$ However, these reviews also serve to highlight the absence of significant improvements in body image in several studies. Thus, despite the existence of a wide range of psychosocial interventions for body image, the authors of existing reviews emphasise the need for more research in this field. ${ }^{12} 13$

When designing and investigating the efficacy (or effectiveness) of psychosocial interventions for body image targeting women diagnosed with breast cancer, ensuring feasibility and acceptability of the intervention is critically important. Ultimately, if an intervention is not feasible or acceptable to women, they are unlikely to access and adhere to the intervention, thereby directly affecting efficacy (or effectiveness). Indeed, poor adherence rates can lead to highly efficacious (or effective) interventions having small effects. As such, reporting guidelines for interventions (eg, Consolidated Standards of Reporting Trials (CONSORT) statement) ${ }^{15}$ recommend to report feasibility and acceptability data such as adherence to the prescribed dosage. Further, seeing as the development of many psychosocial interventions has been led by researchers who may lack insight into endusers' perspectives, it is necessary to assess the evidence for feasibility and acceptability of psychosocial interventions for body image targeting women diagnosed with breast cancer to ensure existing interventions can be translated into practice. Yet, no review to date has been conducted to assess this evidence despite it being crucial for effective resource allocation and preparing/delivering subsequent interventions. Indeed, current reviews omitted descriptive feasibility or acceptability data that were included in the reviewed primary studies. This limits conclusions on whether existing interventions have been successfully executed and delivered to participants as planned. It also limits conclusions on the suitability, appropriateness and relevance of existing interventions from the perspective of women diagnosed with breast cancer.
Additionally, existing reviews note a lack of high-quality trials. There are several possible explanations for this, including a lack feasible and acceptable study methods can hinder researchers' ability to optimise the conduct of their trial as a result of problems with recruitment, enrollment, retention and outcome assessments. Given the scarcity of resources, understanding the feasibility of methods employed across studies evaluating psychosocial interventions for body image targeting women diagnosed with breast cancer and ensuring acceptance is paramount to ensure all evaluative processes work and run smoothly. Thus, a systematic review is planned to assess the evidence for feasibility and acceptability of psychosocial interventions for body image targeting women diagnosed with breast cancer, as well as methods employed in studies conducted to evaluate such interventions. The results of such a review can help not only generate recommendations for practice, but can also help to develop future interventions and evaluations of those interventions. Specifically, results can inform the development of strategies for designing interventions and evaluative studies that are feasible in different contexts and generate acceptance from end-users across a range of settings.

In accordance with good research practice, the purpose of this paper is to describe the protocol for this systematic review in order to outline the rationale for the review, its objectives and the review methods in enough detail that would allow readers to conduct the review and arrive at comparable conclusions. In addition, publishing the protocol enhances transparency, prevents selective reporting of the research outcomes, prevents unnecessary duplication and facilitates subsequent publication of results (as it shows that the methods have been peer-reviewed). ${ }^{16}$

\section{METHODS AND ANALYSIS \\ Protocol and registration}

The protocol for this review is reported in accordance with the Preferred Reporting Items for Systematic Review and Meta-Analysis-Protocols (PRISMA-P) ${ }^{17}$ recommendations, and has been registered in the Prospective Register of Systematic Reviews international registry (ID: CRD42021269062, 11 September 2021). The completed PRISMA-P checklist is presented in Supplemental file 1. Any important protocol amendments will be reported when publishing the results. This systematic review will be performed and reported according to the PRISMA guidelines. $^{18}$

\section{Research question and search methods \\ Research question}

When formulating the research question to guide various aspects of the review process (eg, determining eligibility criteria, search strategy), the PICO research question development tool was used to ensure main concepts were included. The PICO tool focuses on the Population, Intervention (or exposure), Comparison (or 
control) and Outcome(s). However, given this review is not focused on comparison of an intervention and considering quasi-experimental studies lacking a comparison group are eligible, concepts relating to Comparison are not included. The specific research question used is: What is the evidence for feasibility and acceptability (Outcomes) of psychosocial interventions for body image (Intervention) targeting women diagnosed with breast cancer (Population), as well as methods employed in studies conducted to evaluate such interventions.

\section{Electronic databases}

Five electronic databases have been systematically searched for articles published in the English language: Medical Literature Analysis and Retrieval System Online (MEDLINE), Cumulative Index to Nursing and Allied Health Literature, Cochrane Central Register of Controlled Trials, PsychINFO and Excerpta Medica database (EMBASE). To undertake the database searches, a sensitive search strategy was developed drawing on Medical Subject Headings terms and keywords (with appropriate truncations) that have been used in published reviews ${ }^{12} 13$ covering the: (1) target population (ie, women who received a breast cancer diagnosis, including those awaiting treatment, currently receiving treatment, treated with curative intent and living disease-free or living with advanced breast cancer), (2) intervention (ie, psychosocial) and (3) intervention outcome (ie, body image). For the purpose of this review, psychosocial interventions are those focused on psychological, behavioural or social factors. Examples include: psychotherapy, psychoeducation, cognitive-behavioural therapy, cognitive dissonance, self-compassion, peer discussion/support, mindfulness, physical activity/exercise, art therapy and cosmetic/ beauty workshops. Similar to Paterson $e t a l^{3}$ and Davis et $a l^{19}$ an a priori restriction was placed on time period of publication (ie, 2000 onwards) because the year 2000 marks a period of time when the treatment of breast cancer changed. ${ }^{20}$ Further, 'feasibility' and 'acceptability' were omitted from the search strategy because their inclusion could reduce the number of citations identified, and consequently fail to retrieve articles reporting on feasibility and acceptability aspects without using these terms. All quantitative, qualitative or mixed methods studies are eligible for inclusion, and although no specification are made for study design, articles are required to have reported on an intervention evaluation. Articles with mixed cancer populations or couples and articles where psychosocial interventions reflect only a minor part of the intervention are not eligible. The search strategy was pilot tested and finalised in MEDLINE before being translated for use in the four other databases. The search strategy for all included databases is available in Supplemental file 2.

The database searches took place in June 2021, after which point the titles and abstracts of the articles retrieved were imported into Covidence-an internetbased software programme that facilitates collaboration among reviewers during the selection process. ${ }^{21}$ After deduplication of the same records, two reviewers started screening titles and abstracts independently to identify articles eligible for full-text review based on predefined eligibility criteria. For the articles not satisfying eligibility criteria, an exclusion justification code is allotted. This task is currently ongoing. Thereafter, the full-text of articles meeting eligibility criteria, or for which more information is needed to include or exclude, will be assessed independently by two reviewers against eligibility criteria. For articles with insufficient details to determine final eligibility, further details will be sought by emailing the corresponding author (a maximum of two times) to determine their eligibility. Again, for the articles not satisfying eligibility criteria, an exclusion justification code will be allotted. Throughout the screening process, any instances of uncertainty or discrepancy in the classification of articles will be discussed and resolved by discussion.

\section{Manual searches}

Concurrently, the reference list of key articles and reviews retrieved during the database searches will be handsearched to ensure all relevant articles are identified. Additionally, while protocols describing an intervention/ trial without any evaluation are not eligible, steps will be taken to determine if the corresponding data have been published.

\section{Inclusion and exclusion criteria}

A predefined set of eligibility criteria will be used to screen studies for inclusion in this review. Table 1 provides inclusion and exclusion criteria.

\section{Data extraction}

Data from articles included in this review will be extracted independently by two reviewers using a data extraction form developed by the authors. The data extraction form will be piloted on a sample of included studies (20\%) to ensure that all relevant information is captured and there is consistency in data extraction. As well, the reviewers will confer to promote consistency in the interpretation of data extracted, and a third reviewer will verify the accuracy of the data extracted. Data pertaining to study, population, intervention, additional information and outcomes will be extracted and managed using Microsoft Excel (Microsoft Corporation, Seattle, Washington, USA). Any uncertainties will be resolved by discussion. For study, the following data will be extracted: first author, country where the study took place, year of publication, study design, study aims/objectives/purposes, multisite (yes/ no; if yes, number of sites) and recruitment strategy(ies).

For population, the following data will be extracted: eligibility criteria, sample size at baseline/start of the study (total and per group), participants' age (mean, SD and range (total sample and per group)), socioeconomic status (eg, income, education), comorbidities, body image at baseline/start of the study, anthropometrics at baseline/start of the study (eg, body mass index, body 
Table 1 Inclusion and exclusion criteria for the selection of studies.

\begin{tabular}{|c|c|c|}
\hline Characteristics & Included & Excluded \\
\hline Publication type & $\begin{array}{l}\text { Peer-reviewed } \\
\text { English-language } \\
\text { Published from } 2000 \text { to June } 2021\end{array}$ & $\begin{array}{l}\text { Non-English-language articles } \\
\text { Opinion pieces (eg, editorials/commentaries, } \\
\text { letters to editor), reviews/meta-analyses, } \\
\text { letters, practice guidelines, protocols* } \\
\text { Grey literature (defined as any print or } \\
\text { electronic literature that is produced by } \\
\text { government, academia, business and } \\
\text { industry, and is not controlled by commercial } \\
\text { publishers; }{ }^{28} \text { this includes dissertations/ } \\
\text { theses, conference abstracts/proceedings, } \\
\text { book chapters, unpublished manuscript, } \\
\text { unpublished data, government and agency } \\
\text { reports) }\end{array}$ \\
\hline Study population & $\begin{array}{l}\text { Women ( } \geq 18 \text { years of age) } \dagger \text { diagnosed with breast } \\
\text { cancer (including ductal carcinoma in situ or metastatic } \\
\text { disease), either awaiting treatment, currently receiving } \\
\text { treatment, treated with curative intent and living } \\
\text { disease-free or living with advanced breast cancer }\end{array}$ & $\begin{array}{l}\text { Children/adolescents (<18years) } \\
\text { Men } \\
\text { Women diagnosed with other types of } \\
\text { cancer } \\
\text { Mixed cancer samples }\end{array}$ \\
\hline Intervention & $\begin{array}{l}\text { Any community-basedł psychosocial intervention, } \\
\text { supervised or unsupervised, designed to effect change } \\
\text { in body image } \\
\text { Interventions delivered remotely via telephone, } \\
\text { messaging, or digital technology (eg, online), face-to- } \\
\text { face or that blend digital and human support }\end{array}$ & $\begin{array}{l}\text { Pharmacological interventions } \\
\text { Surgical interventions } \\
\text { Couples interventions }\end{array}$ \\
\hline
\end{tabular}

${ }^{* a}$ Protocols describing an intervention/trial without any evaluation are not eligible; however, steps will be taken to determine if the corresponding data have been published. Articles evaluating the concept of psychosocial interventions for body image in women diagnosed with breast cancer or studies describing the initial development of an intervention are also ineligible.

†The mean age of the sample is required to fall $\geq 18$ years.

$\ddagger$ Defined as an intervention delivered to women residing in the community rather than in a hospital or other healthcare facility (eg, rehabilitation centre). This said, interventions may have been delivered at a hospital or other healthcare facility.

circumference (waist, hips, limbs), skinfold thickness, body fat percentage), physical activity level at baseline/ start of the study, stage of cancer, type(s) of cancer treatments (with frequencies/percentages) and treatment status at baseline/start of the study (on/off/mixed).

For intervention, the following data will be extracted: type (eg, psychotherapy, psychoeducation, psychological intervention/support, behavioural intervention/support, cognitive behavioural therapy, peer intervention/support, physical activity/exercise (eg, resistance, weight, strength, endurance, interval, highintensity, aerobic, anaerobic, gravity resistive, isotonic and isometric exercise/training, walking, yoga), counselling, cognitive dissonance, education, self-help, expressive or creative writing, mindfulness, guided imagery, camouflage-based or cosmetic-based (eg, concealing or altering appearance such as make-up workshops)), theoretical basis/approach(es) (yes/no; if yes, describe), dosage (number, frequency and length of sessions, duration of intervention), format (eg, face-to-face, remote), setting (eg, online, home-based, hospital-based, outpatient clinic, community organisation/centre), timing (ie, before, during or after treatment, mixed), intervention workforce (ie, number of person(s) delivering the intervention, their profession (eg, psychiatrist, fitness professional, clinical psychologist, author, student), training received to deliver the intervention (eg, timing, procedures, training conducted by who)), modality (ie, group-based, individual, mixed) and control/comparison group(s) (yes/ 
no; if yes, describe (eg, no intervention (usual care), waitlist, alternative intervention)).

For additional information, the following data will be extracted: participant-blinding (yes/no/not applicable), assessor-blinding (yes/no/not applicable), intentionto-treat analyses (yes/no/not applicable), differential attrition between groups (yes/no/not applicable; if yes, describe), sample size/power calculation were reported (yes/no), guideline(s) or checklist(s) were used for reporting (yes/no; if yes, describe) and trial registration (yes/no; if yes, where).

For outcomes, conceptual definitions of feasibility and acceptability within the literature vary considerably, underscoring the ambiguity of these concepts. Consequently, there are inconsistencies in the way feasibility and acceptability have been operationalised and reported. To allow for an in-depth discussion about the feasibility and acceptability of study methods and interventions, no one measure of feasibility and acceptability will be targeted. Rather, several measures of feasibility and acceptability will be targeted, including items from the CONSORT 2010 statement,${ }^{15}$ to establish whether the study methods (ie, design and procedures) and intervention: (1) can be successfully executed by the researchers and delivered to the participants as planned (feasibility) and (2) are suitable to participants (acceptability). ${ }^{22}$ Accordingly, the following data will be extracted for feasibility and acceptability of the study methods: (1) number of recruiting centres and recruitment duration, (2) number of persons assessed for eligibility and reasons for exclusion (ie, not meeting inclusion criteria, declined to participate, other reasons), (3) number of participants randomised and reasons for refusal, (4) number of participants allocated to the intervention(s), who received the allocated intervention(s), and who did not received the allocated intervention(s) and reasons, (5) number of participants lost to follow-up (ie, who did not complete follow-up assessments) and reasons, (6) number of participants analysed, and number excluded from analysis and reasons and (7) other quantitative or qualitative data related to feasibility and acceptability of the study methods (eg, number of persons who consented and reasons for refusal, qualitative data directly related to women's opinions of the study methods (eg, randomisation, study staff interactions, frequency of assessments, measures used, modality of assessments)).

In addition, the following data will be extracted for feasibility and acceptability of the intervention: (1) participants' satisfaction ratings/scores, (2) percentage of participants who attended all or a certain percentage of the prescribed sessions, (3) percentage of participants who attended all or a certain percentage of the prescribed session duration, (4) number of participants who discontinued the intervention(s) and reasons, (5) number and type(s) of adverse events related to the intervention(s) and (6) other quantitative or qualitative data related to feasibility and acceptability of the intervention(s) (eg, qualitative data directly related to women's opinions of the content, dosage or mode of delivery of the intervention, as well as the person(s) delivering the intervention).

Finally, while the proposed review is not designed to assess the efficacy or effectiveness of interventions seeking to improve body image, it may be especially important to synthesise body image measures used as these may impact the feasibility and acceptability of the study methods. Thus, any measure reasonably perceived to represent some aspect of women's body-related thoughts, perceptions, attitudes, beliefs, feelings and/or behaviours (eg, body dissatisfaction/satisfaction, body/physical appreciation/acceptance, appearance/shape/weight concern/ distress, body/physical self-concept/worth/esteem, embodiment, self-image, social physique anxiety, social/ appearance comparison, self-objectification, thin-ideal internalisation) will be considered relevant to this review, and accordingly, information on the measure will be extracted alongside the number and timing of administration (eg, preintervention, mid-intervention, postintervention, follow-up(s)). Reviewers will refer to a list of 88 measures previously used in studies on body image with women diagnosed with breast cancer to inform their extraction. $^{23}$

Of note, in cases where information is not explicitly reported, but can be estimated, it will be. If, however, the data reported in the published articles are absent (or unclear), the corresponding author will be contacted by email to obtain further details. Two such attempts will be made. If there is no response from the corresponding author, the data not available within the article will be marked as 'not available'.

\section{Quality assessment}

Two reviewers will conduct quality assessment using the Mixed Methods Appraisal Tool (MMAT, V.2018) ${ }^{24}$ for reviews including qualitative, quantitative and mixed methods studies using different study designs. The MMAT facilitates critical appraisal of studies within systematic reviews by providing methodological quality criteria for different study designs/methods (such as those that will be reviewed) within a single tool. Similar to the Cochrane collaboration's tool for assessing risk of bias in randomised trials, ${ }^{25}$ the MMAT covers items that may hinder the validity of the findings of a study. Disagreement will be resolved by mutual consensus or discussion with a third reviewer. Following recommendations, ${ }^{24}$ appraisal will be discussed qualitatively and quantitative quality scores will not be derived. Moreover, study quality will not be used as a criterion for inclusion; rather, it will be used to guide understanding of the relative strength of the data.

\section{Data synthesis}

The search and selection process based on these criteria will be presented in a flow diagram according to the PRISMA statement. Owing to the wide array of studies and interventions reported in previous reviews, it is anticipated that a quantitative synthesis of studies reporting quantitative data will be neither feasible nor informative. 
Accordingly, no meta-analysis will be performed; instead, quantitative data for feasibility and acceptability will be summarised narratively. For qualitative data, thematic analysis will be undertaken. This will comprise three steps: (1) free coding of data from included studies, (2) organisation of free codes into related areas or 'descriptive themes' and (3) inferring 'analytical' themes that go beyond the findings of the original studies. ${ }^{26}{ }^{27}$ Coding will be inductive. Assessment of meta-bias will not be performed.

\section{Patient and public involvement}

Patients and the public were not involved in the design and conduct of this review. During the dissemination phase of this project, the findings will be discussed with members of the public who will be asked for their thoughts on how best to share results to others (eg, newsletter, social media) to encourage and support patient/ public involvement.

\section{ETHICS AND DISSEMINATION}

No ethical approval is required because this is a protocol for a systematic review. Upon completion, results will be submitted for publication in a peer-reviewed scientific journal and for presentation at a relevant conference.

\section{DISCUSSION}

The provision of psychosocial interventions for body image targeting women diagnosed with breast cancer is a rapidly expanding area of research and practice to improve cancer care. Current intervention approaches include: psychoeducation, cognitive behavioural therapy, physical activity, art therapy, mindfulness, and expressive writing. ${ }^{12-14}$ Existing reviews of such interventions focus on their effects on women's body image, which is useful to provide insight into whether psychosocial interventions for body image targeting women diagnosed with breast cancer are efficacious (or effective). Nevertheless, reviews assessing the feasibility and acceptability of interventions remain equally important because, ultimately, if an intervention is not feasible or acceptable to women, they are unlikely to access it and adhere to it, thereby directly affecting its effects on body image. Although previous reviews have presented data on the types and efficacy of psychosocial interventions for body image, ${ }^{12} 13$ to our knowledge, this will be the first review to synthesize evidence on the feasibility and acceptability of such interventions, as well as study methods used to evaluate them. The findings can help identify key aspects of study methods (eg, designs, procedures) and psychosocial interventions that have been most feasible and acceptable. This information will help guide the development, implementation, and evaluation of psychosocial interventions for body image targeting women diagnosed with breast cancer. This review will also help identify gaps in the existing literature to provide direction for future research.

This review has several notable strengths. First, the protocol is being published to provide detailed, concise, and transparent descriptions of the review methods, enhance transparency, prevent selective reporting of the research outcomes, prevent unnecessary duplication, and facilitate subsequent publication of results. Second, search methods (eg, range of databases, search strategy) are comprehensive to ensure eligible studies are retrieved and included. Third, all experimental studies using quantitative, qualitative and mixed methods that were published in the year 2000 onwards are eligible for inclusion to enable a broad and in-depth analysis of existing interventions. Additionally, data extraction will be conducted systematically with the use of a piloted data extraction form and corresponding authors will be contacted for further details.

Nevertheless, there are foreseeable limitations to this review due to the developing status of this field of research. First, many studies have not primarily aimed to explore feasibility or acceptability, and thus may have limited information on the feasibility and/or acceptability of the study methods and/or intervention. Relatedly, it may not be possible to draw conclusions about the influence of various factors (eg, sociodemographic, clinical) on the feasibility and acceptability of the study methods and/or intervention. Second, the concepts of 'feasibility' and 'acceptability' are complex and there is a lack of consistency in their definition and interpretation, which may present challenges while interpreting the evidence and summarising it across studies. Nevertheless, the development of guidelines structuring the reporting of intervention studies like the CONSORT statement ${ }^{15}$ has likely improved the monitoring and reporting of such data over time, and attempts to synthesize it are warranted to facilitate the implementation of effective interventions on a larger scale. Third, the small sample sizes in many studies may limit the generalizability of the findings. Fourth, the requirement for studies to be published in the English language may result in a biased sample, potentially excluding relevant articles if published in other languages, though no geographic restrictions will be imposed. Last, this review is restricted to peer-reviewed articles; grey literature searches will not be conducted. Using traditional electronic databases to search the literature may not identify all relevant studies and introduce bias such as publication and time-lag bias. Nevertheless, other means of searching for articles (eg, handsearching, contacting contact persons of identified protocols to determine if the corresponding data have been published) will be taken.

In ending, the development of psychosocial interventions for body image targeting women with breast cancer is a burgeoning field of study and the evidence base will likely grow quickly. As more research focuses on developing, implementing and evaluating such interventions, failure to take into consideration feasibility and 
acceptability aspects of studies methods and interventions based on previously published articles may not only serve as a barrier to the conduct of high-quality trials, but may threaten sustainability and disregard the perspective of end-users (notably women receiving the intervention). Future studies will benefit from a summary of evidence on the feasibility and acceptability of psychosocial interventions for body image targeting women with breast cancer, as well as study methods used to evaluate them, which may provide guidance on how to adapt study designs, procedures, and interventions in various settings. It is therefore believed that this systematic review is timely and will make a valuable contribution to fill an existing knowledge gap.

Acknowledgements The first author holds a Tier II Canada Research Chair in Physical Activity Promotion for Cancer Prevention and Survivorship. This manuscript was written while the second author was supported by a Joseph-Armand Bombardier Canada Graduate Doctoral Scholarship, and the third author by a salary award from the Fonds de recherche du Québec - Santé.

Contributors JB (corresponding author) is the overall project lead. She conceptualised the review, created the review design, advised on the search strategy and screening process, and wrote the first draft of the protocol manuscript. JP participated in the review design, created and executed the search strategy, and critically revised the manuscript. $A B$ advised on the quality assessment approach and data extraction form, and critically revised the manuscript. All authors approved the final version of this manuscript.

Funding This study was supported by an Insight Grant from the Social Sciences and Humanities Research Council of Canada. Grant number: N/A (to be released).

Competing interests None declared.

Patient consent for publication Not applicable.

Supplemental material This content has been supplied by the author(s). It has not been vetted by BMJ Publishing Group Limited (BMJ) and may not have been peer-reviewed. Any opinions or recommendations discussed are solely those of the author(s) and are not endorsed by BMJ. BMJ disclaims all liability and responsibility arising from any reliance placed on the content. Where the content includes any translated material, BMJ does not warrant the accuracy and reliability of the translations (including but not limited to local regulations, clinical guidelines, terminology, drug names and drug dosages), and is not responsible for any error and/or omissions arising from translation and adaptation or otherwise.

Open access This is an open access article distributed in accordance with the Creative Commons Attribution Non Commercial (CC BY-NC 4.0) license, which permits others to distribute, remix, adapt, build upon this work non-commercially, and license their derivative works on different terms, provided the original work is properly cited, appropriate credit is given, any changes made indicated, and the use is non-commercial. See: http://creativecommons.org/licenses/by-nc/4.0/.

\section{ORCID iD}

Jennifer Brunet http://orcid.org/0000-0003-3242-5444

\section{REFERENCES}

1 Sung H, Ferlay J, Siegel RL, et al. Global cancer statistics 2020: GLOBOCAN estimates of incidence and mortality worldwide for 36 cancers in 185 countries. CA Cancer J Clin 2021;71:209-49.

2 Gradishar WJ, Anderson BO, Abraham J, et al. Breast cancer, version 3.2020, NCCN clinical practice guidelines in oncology. J Natl Compr Canc Netw 2020;18:452-78.

3 Paterson CL, Lengacher CA, Donovan KA, et al. Body image in younger breast cancer survivors: a systematic review. Cancer Nurs 2016;39:E39-58.
4 Begovic-Juhant A, Chmielewski A, Iwuagwu S, et al. Impact of body image on depression and quality of life among women with breast cancer. J Psychosoc Oncol 2012;30:446-60.

5 Mokhatri-Hesari P, Montazeri A. Health-related quality of life in breast cancer patients: review of reviews from 2008 to 2018. Health Qual Life Outcomes 2020;18:338.

6 Tripathi L, Datta SS, Agrawal SK, et al. Stigma perceived by women following surgery for breast cancer. Indian J Med Paediatr Oncol 2017;38:146-52.

7 Kang NE, Kim HY, Kim JY, et al. Relationship between cancer stigma, social support, coping strategies and psychosocial adjustment among breast cancer survivors. J Clin Nurs 2020;29:4368-78.

8 Esser P, Mehnert A, Johansen C, et al. Body image mediates the effect of cancer-related stigmatization on depression: a new target for intervention. Psychooncology 2018;27:193-8.

9 Gonzalez BD, Jacobsen PB. Depression in lung cancer patients: the role of perceived stigma. Psychooncology 2012;21:239-46.

10 Phelan SM, Griffin JM, Jackson GL, et al. Stigma, perceived blame, self-blame, and depressive symptoms in men with colorectal cancer. Psychooncology 2013;22:65-73.

11 Esplen MJ, Fingeret MC. Body image-An important dimension in cancer care. In: Breitbard W, Butow P, Jacobsen P, et al, eds. Psycho-Oncology. 4 ed. New York, NY: Oxford University Press, 2021: 303-12.

12 Lewis-Smith H, Diedrichs PC, Rumsey N, et al. Efficacy of psychosocial and physical activity-based interventions to improve body image among women treated for breast cancer: a systematic review. Psychooncology 2018;27:2687-99.

13 Sebri V, Durosini I, Triberti S, et al. The efficacy of psychological intervention on body image in breast cancer patients and survivors: a systematic-review and meta-analysis. Front Psychol 2021;12:611954.

14 Effa CJ, Dolgoy ND, McNeely ML. Resistance exercise and art therapy on body image in breast cancer: a scoping review. Womens Health Rep 2020;1:424-35.

15 Schulz KF, Altman DG, Moher D. Consort 2010 statement: updated guidelines for reporting parallel group randomised trials. BMC Med 2010;340:c332.

16 JMIR. Why should I publish my protocol or grant proposal? 2021. Available: https://support.jmir.org/hc/en-us/articles/115002860428 [Accessed June 2021].

17 Moher D, Shamseer L, Clarke M, et al. Preferred reporting items for systematic review and meta-analysis protocols (PRISMA-P) 2015 statement. Syst Rev 2015;4:1.

18 Moher D, Liberati A, Tetzlaff J, et al. Preferred reporting items for systematic reviews and meta-analyses: the PRISMA statement. PLoS Med 2009;6:e1000097.

19 Davis C, Tami P, Ramsay D, et al. Body image in older breast cancer survivors: a systematic review. Psychooncology 2020;29:823-32.

20 Park J-H, Anderson WF, Gail MH. Improvements in US breast cancer survival and proportion explained by tumor size and estrogenreceptor status. J Clin Oncol 2015;33:2870-6.

21 Covidence. Veritas health innovation Covidence systematic review software. Melbourne, Australia, 2021.

22 Feeley N, Cossette S, Côté J, et al. The importance of piloting an RCT intervention. Can J Nurs Res 2009;41:85-99.

23 Brunet J, Price J. A scoping review of measures used to assess body image in women with breast cancer. Psychooncology 2021;30:669-80.

24 Hong QN, Pluye P, Fàbregues S, et al. Improving the content validity of the mixed methods appraisal tool: a modified e-Delphi study. $J$ Clin Epidemiol 2019;111:49-59.

25 Higgins JPT, Altman DG, Gøtzsche PC, et al. The Cochrane collaboration's tool for assessing risk of bias in randomised trials. BMJ 2011;343:d5928.

26 Thomas J, Harden A. Methods for the thematic synthesis of qualitative research in systematic reviews. BMC Med Res Methodol 2008;8:45.

27 Suri $\mathrm{H}$, Clarke D. Advancements in research synthesis methods: from a methodologically inclusive perspective. Rev Educ Res 2009;79:395-430.

28 Auger CP. Information sources in grey literature. New Providence, NJ: Bowker-Saur, 1998. 